\title{
Crocin regulates the proliferation and migration of neural stem cells after cerebral ischemia by activating the Notch1 pathway
}

\author{
Baizhu An ${ }^{1}$, Ying $\mathrm{Ma}^{2}$, Yongliang $\mathrm{Xu}^{1}$, Xing Liu${ }^{1}$, Xudong Zhang ${ }^{3}$, Jing Zhang ${ }^{1}$, Chunzhuang Yang ${ }^{1}$ \\ ${ }^{1}$ Department of Anatomy, Mudanjiang Medical University, Mudanjiang, China, ${ }^{2}$ Department of Library, Mudanjiang Medical \\ University, Mudanjiang, China, ${ }^{3}$ Department of Physiology, Mudanjiang Medical University, Mudanjiang, China
}

\begin{abstract}
Aim of the study: To investigate the effects of crocin on proliferation and migration of endogenous neural stem cells and the Notch1 signalling pathway in rats after cerebral ischemia reperfusion.

Material and methods: SD rats were randomly divided into the sham operation group, model group and administration group (crocin). Middle cerebral artery occlusion (MCAO/R) was used to establish the focal cerebral ischemia reperfusion model in rat. After surgical treatment, the treatment group was treated with crocin. Quantitative polymerase chain reaction ( $($ PCR) was used to detect the changes in the expression of Notch1, Bax and bcl-2 proteins in rat endogenous neural stem cells after cerebral ischemia reperfusion. ELISA was used to detect changes in inflammatory factors. Neural stem cells were cultured in vitro, which were divided into: the normal control group, the hypoglycaemic deprivation/reoxygenation group, hypoglycaemic deprivation/reoxygenation group with a low concentration of crocin, and hypoglycaemic deprivation/reoxygenation group with a high concentration of crocin. The cell proliferation assay detects cell activity. The cell migration assay tests the cell migration ability. And flow cytometry was used to determine cell apoptosis.

Results: Compared with the sham group, the Notch1 signalling pathway was activated in the model group. The expression of Notch1 in the crocin group was increased compared to the model group. Crocin can inhibit the release of inflammatory factors. The results of our experiments showed that crocin could induce the proliferation and migration of neural stem cells and inhibit the apoptosis of neural stem cells in the hypoglycaemic/reoxygenation model group.

Conclusions: Crocin sufficiently promotes the proliferation and migration of neural stem cells and inhibits the apoptosis of these cells in rats after ischemia-reperfusion by manipulating the Notch signalling pathway.
\end{abstract}

Key words: crocin, MCAO/R, OGD/R, nerve regeneration, Notch1.

\section{Introduction}

Cerebral ischemia is known as a common acute cerebrovascular disease with a high occurrence of disability and mortality $[25,27]$. The key of treatment is to prevent functional loss of neurons due to ischemic penumbra and to promote the recovery of neuronal functions after injury. Although important advances have been made in hyper-early thrombolysis and neu-

Communicating author

Chunzhuang Yang, Department of Anatomy, Mudanjiang Medical University, Mudanjiang 157011, China,

e-mail: Chunzhuangyang412@163.com 
ronal protection in the acute phase, neuronal death is inevitable in most patients with cerebral ischemia. How to promote the recovery of neuronal function in the convalescent period of cerebral ischemia is still the focus of current research on cerebral ischemia. Neural stem cells, a key component in the nervous system, are known to differentiate into neurons and glial cells. Thus, they provide an opportunity to elevate the loss of neurons and the restoration of neural stem cell function is therefore important for the treatment of cerebral ischemia $[2,20,24]$.

Recent studies have found that neural stem cells proliferate and differentiate after cerebral ischemia, indicating their participation in the physiological and pathological process of cerebral ischemia. Rat cerebral ischemia model established by Kee et al. [13] used two-vessel occlusion combined with hypotension and they found an increased proliferation of dentate gyrus stems cells by 2 3 times after 1 week of ischemia reperfusion. And 2 weeks later, $60 \%$ of the proliferating cells expressed CRMP4, a marker indicating neurons in their naive stage. After 5 weeks of reperfusion, $36 \%$ of the proliferating cells became matured neurons. Jin et al. [11] found that cerebral ischemia not only promotes the proliferation of neural stem cells in the dentate gyrus of the hippocampus, but also affects the neural stem cells of subventricular zone (SVZ) in the inferior lateral ventricle. Moreover, the proliferation of neural stem cells in the un ischemic/non-ischemic side of the cerebral hemisphere also increased, and the proliferation of neural stem cells in the ischemic side of the dentate gyrus (DG) was the most obvious. Arvidsson et al. [1] compared the effects of middle cerebral artery occlusion (MCAO) for 30 min and 2 hours on neural stem cells, and the results showed that cerebral ischemia for 30 min only caused mild and variable stem cell proliferation. After cerebral ischemia for 2 hours, DG neural stem cells were significantly proliferated, and this increase in proliferation was not dependent on the death of hippocampal neurons, nor was it related to the degree of damage to the cerebral cortex. However, the relationship between ischemia and stem cells is not completely linear, and severe ischemia may lead to a decrease in stem cells. Therefore, it is of great significance to identify the key pathways in the process of cerebral ischemia, and timely intervene in the cerebral ischemia injury with drugs to promote the repair of neural stem cells.

The Notch cell signalling pathway contains Notch receptor, ligand (DSL), CSL protein and downstream target gene $[28,30,32]$. The activation process of a typical Notch signalling pathway involves the binding of Notch receptor to the ligand DSL, and subsequently the released Notch intracellular domain (NICD) which then translocates into the nucleus and binds to the CSL protein to activate the downstream targets $[18,19]$. Studies have shown that the Notch signalling pathway regulates the proliferation and differentiation of neural stem cells $[3,6]$. The Notch signalling pathway can interact with the receptor through the ligand of adjacent cells to transmit signals and further play an important signalling pathway of its biological function. This pathway is involved in the process of maintenance and differentiation of neural stem cells with its control of variety of neural stem cell functions. The crocin is a perennial iris crocus (Crocus sativus L.) Dry stigma [12]. Crocin (crocin) is a series of ester glycosides formed by the combination of crocin acid and different sugar, a kind of water-soluble carotenoid compound in crocin [35]. Crocin has a protective effect on cerebral ischemia-reperfusion injury, but whether crocin can regulate the Notch1 signalling pathway and promote the proliferation and migration of neural stem cells remains to be investigated.

In this study, the effect of crocin on the Notch signalling pathway of nerve cells after cerebral ischemia reperfusion was observed, and the mechanism of crocin in treating ischemic stroke was discussed. Meanwhile, oxygen-glucose deprivation/reoxygenation $(\mathrm{OGD} / \mathrm{R})$ was used to simulate cerebral ischemia-reperfusion in vivo to observe the effects of crocin on the proliferation, vitality and Notch1 signalling pathway of neural stem cells. In order to find a new therapeutic target for the mitigation or even prevention of cerebral ischemia.

\section{Material and methods \\ Experimental animals}

Female SD rats (300 \pm 20 g) were provided by Hunan Tianqin, a biotechnology company and kept in 12 hours day/night cycles. All conducted procedures were approved by the Ethics Committee of Mudanjiang Medical University.

\section{MCAO/R animal model}

According to the random number table, the rats were randomly divided into groups, and the left middle cerebral artery occlusion (MCAO) model was con- 
structed by a line bolting method $[4,15,29]$. The specific operation was as follows: the neck skin of the rats was lengthwise incised about $1.5 \mathrm{~cm}$ to the left of the anterior median line. The left common carotid artery (LCCA), external carotid artery (ECA) and internal carotid artery (ICA) were isolated by avoiding the blood vessels. The ECA and LCCA were ligated with thread. Near bifurcation of LCCA (the fixed distance is 2-3 mm), a small cut with ophthalmic shear was made, and the MCAO plug line was inserted into the opening slowly. When the plug line reached $20 \mathrm{~mm}$ near the bifurcation of LCCA, the speed was slowed down and a little resistance was felt, indicating that the plug line has blocked the middle cerebral artery. The plug line and ICA was clamped to prevent the plug line from slipping out. After 120 min, the artery clip was opened and the thrombus line was carefully extracted to achieve the goal of ischemia-side reperfusion. ICA was subsequently ligated on the operative side. Penicillin disinfection and suture incision was done. Before the operation, the rats fasted for 12 hours and drank water freely. In the sham operation group, the left cervical blood vessels were isolated, but the embolus was not inserted. The concentration of crocin in the low concentration group was $10 \mathrm{mg} / \mathrm{kg}$, and that of the high concentration group was $50 \mathrm{mg} / \mathrm{kg}$, 1 time/day. The sham operation group and model group were given the same amount of distilled water, 1 time/day. All the rats were anesthetized by administration of chloral hydrate $(10 \% \mathrm{w} / \mathrm{v})$ intraperitoneally (i.p.).

\section{Cell culture}

Neural stem cell complete medium $+10 \%$ FBS (SH30088.03, Hyclone, USA) was used to adjust the density of neural stem cells to $2 \times 10^{5} / \mathrm{ml}$. Cell culture medium DMEM/F12 (1 : 1), 11320-033, Gibco, USA. $20 \mu$ l of neural stem cells were inoculated into 96-well plates coated with polylysine, and cultured in an incubator for 2 hours to promote adherence. Then $50 \mu \mathrm{l}$ neural stem cells complete medium was added to continue culturing. In the experiment, the normal control group only added $10 \%$ normal rat serum to the complete medium, and the OGD/R group as the control continued to be cultured with neural stem cell complete medium containing $10 \%$ normal rat serum after reoxygenation. In the crocin group, $10 \mu \mathrm{M}$ and $50 \mu \mathrm{M}$ crocin were added to the complete culture medium of neural stem cells.

\section{Establishment of OGD/R neural stem cell culture model}

The culture medium of the neural stem cells to be tested was changed to the artificial cerebrospinal fluid without sugar in a pre-balanced mixture of $5 \%$ $\mathrm{CO}_{2}$ and $95 \% \mathrm{~N}_{2}$ for 1 hour. Its composition ( $\mathrm{mmol} / \mathrm{l}$ ) was as follows: $\mathrm{NaCl}: 123, \mathrm{KCl}: 3.75, \mathrm{KH}_{2} \mathrm{PO}_{4}: 1.25$, $\mathrm{NaHCO}_{3}: 26, \mathrm{MgCl}: 21, \mathrm{CaCl}_{2}: 2, \mathrm{pH}=7.4$. The fluid was put in a closed constant temperature cultivation device (COOK desktop incubator, model: COOKKMINC-1000, origin: Australia), the temperature was maintained at $35^{\circ} \mathrm{C}$, the culture gas was changed to $5 \% \mathrm{CO}_{2}, 95 \% \mathrm{~N}_{2}$ mixed gas. After 3 hours, the artificial cerebrospinal fluid was replaced with the culture medium and placed back in a $37^{\circ} \mathrm{C}$ incubator containing $5 \% \mathrm{CO}_{2}$ for 1 day.

\section{Cell proliferation experiment}

Neural stem cells were seeded in 96-well plates previously coated with polylysine. After the experiment, $20 \mu \mathrm{MTT}$ (5 g/l) was added to each well of the 96-well plate, the cells continued to incubate at $37^{\circ} \mathrm{C}$ for 4 hours, the supernatant was discarded, $150 \mu$ l dimethyl sulfoxide was added to each well, it was shaken for $10 \mathrm{~min}$, the crystal was fully dissolved, $490 \mathrm{~nm}$ Wavelength was selected, the absorbance $(A)$ value of each well was measured on an enzyme-linked immunoassay detector. The data are expressed in relative multiples compared to the normal control group.

\section{Cell migration assay}

Transfected cells were first exposed to OGD/R conditions and then using Transwell migration chambers (8.0 $\mu \mathrm{m}$ pore, Costar-Corning, NY) were used to perform the migration assay. The upper chamber was filled with $100 \mu \mathrm{l}$ suspended cells in serum-free medium, and the lower chamber was filled with complete medium. After incubation at $37^{\circ} \mathrm{C}$ for 24 hours, non-migrated cells on the upper compartment were removed by cotton swabs. The migrated cells were stained with crystal violet (Beyotime, Nantong, China) and quantified under a light microscopy (Nikon, Japan).

\section{Flow cytometry to detect apoptosis}

Cells were seeded in a 6 -well plate at $1 \times 10^{5} /$ well. After the cells adhered to the wall, three repli- 
cate wells were set in each group, and cultured for 48 hours after different treatments. The cells were digested with trypsin without EDTA and centrifuged at $2000 \mathrm{r} / \mathrm{min}$ for $5 \mathrm{~min}$. The cells were washed twice with phosphate-buffered saline (PBS) and re-suspend by adding $500 \mu \mathrm{l}$ of binding buffer. $5 \mu \mathrm{l}$ annexin V-FITC and $10 \mu \mathrm{l}$ PI was added successively, mixed well, incubated at room temperature in the dark for $15 \mathrm{~min}$, the cell number was adjusted to $1 \times 10^{4}$ /tube, and the apoptosis of each group was detected by flow cytometry.

\section{TUNEL detects apoptosis}

The cells were washed with PBS buffer. Then they were fixed with $4 \%$ paraformaldehyde for 30 min. $0.3 \%$ triton $\mathrm{X}-100$ was added to the cells and incubated at room temperature for $5 \mathrm{~min}$. They incubated in $0.3 \%$ hydrogen peroxide solution $\left(0.3 \% \mathrm{H}_{2} \mathrm{O}_{2}\right)$ prepared by PBS at room temperature for $20 \mathrm{~min}$ to inactivate the endogenous peroxidase in sections. Then they were washed with PBS 3 times. An appropriate amount of TUNEL detection fluid was prepared and fully mixed. $50 \mu \mathrm{l}$ TUNEL detection solutions was added to the sample and incubated in the dark at $37^{\circ} \mathrm{C}$ for $60 \mathrm{~min}$, then it was washed with PBS 3 times. Fluorescence microscope was used to observe the film after the film was sealed with anti-fluorescence quenching liquid (Nikon, Japan). The excitation wavelength range used is 450-500 $\mathrm{nm}$ and the emission wavelength range was $515-565 \mathrm{~nm}$.

\section{QPCR}

Cells were seeded in 6-well plates at $1 \times 10^{5}$ cells/ well. After the cells adhered to the cells, they were treated separately according to the experimental group, and an untreated control group was set up at the same time. The cells were collected after 48 hours. The TRIzol method extracted the total RNA of cells. PrimeScript RT Reagent Kit was used to reverse transcribe the total RNA of each cell into cDNA. cDNA was taken for real-time fluorescence quantitative PCR detection. The primers were synthesized by Shanghai Shenggong Biological Engineering Co. The primer sequence was as follows: Bcl-2, F: ATGCCTTTGTGGAACTATATGGC, R: GGTATGCACCCAGAGTGATGC; Bax, F: TGAAGACAGGGGCCTTTT TG, R: AATTCGCCGGAGACACTCG; Notch1, F: TCGTGTGTCAAGCTGATGAGG, R: AGTTCGGCAGCTACAGGT-
CACAA; Hes-1, F: GCAGACATTCTGGAAATGACTGTGA, R: GAGTGCGCACCTCGGTGTTA; $\beta$-actin, F: GGCTGTATTCCCCTCCATCG, R: CCAGTTGGTAACAATGCCATGT. Reaction conditions were as follows: $94^{\circ} \mathrm{C}$ for $2 \mathrm{~min}$; $92^{\circ} \mathrm{C}$ for $20 \mathrm{~s}, 56^{\circ} \mathrm{C}$ for $30 \mathrm{~s}, 72^{\circ} \mathrm{C}$ for $45 \mathrm{~s}$, a total of 30 cycles, the last extension $72^{\circ} \mathrm{C}$ for $7 \mathrm{~min}$. The $2^{-\Delta \Delta \mathrm{ct}}$ method was used to calculate the relative expression of each gene.

\section{ELISA}

Enzyme-linked immunosorbent assay (ELISA) was used to detect the expression of inflammatory factors in the neural stem cell culture. The cells were collected by reference to ELISA. The OD value was detected at the wavelength of $450 \mathrm{~nm}$ with an enzyme marker, and then the OD value of the blank control group was subtracted. Then the concentration of the standard substance and the measured OD value were used as the standard curve to calculate the concentration of the sample to be tested.

\section{Statistical method}

All the counting data were processed by SPSS20.0 statistical software, and the results were expressed as $\mathrm{x} \pm \mathrm{s}$. Independent sample $t$ test was used for comparison between two groups, and one-way ANOVA was used for comparison between multiple groups. $P<0.05$ was considered statistically significant.

\section{Results}

\section{In the MCAO/R model, the Notch1 signalling pathway was up-regulated and the content of inflammatory factors increased}

To study the role of the Notch1 signalling pathway in cerebral ischemia, we first constructed a rat $M C A O / R$ model, and then detected the changes in the expression of Notch1 in the sham group and the model group. We found an increased level of Notch1 in the MCAO/R model group compared to the control (Fig. 1A). Cell surface Notch ligand, namely the DSL (Delta, Serrate, Lag-2) protein family, which includes the mammalian derived jagged ligand 1 (Jagged1) was also up-regulated in the MCAO/R model group (Fig. 1B). Further results showed an elevated level of Hes1 and c-Src in the MCAO/R model group (Fig. 1C, D). Moreover, compared with the control group, the 
A

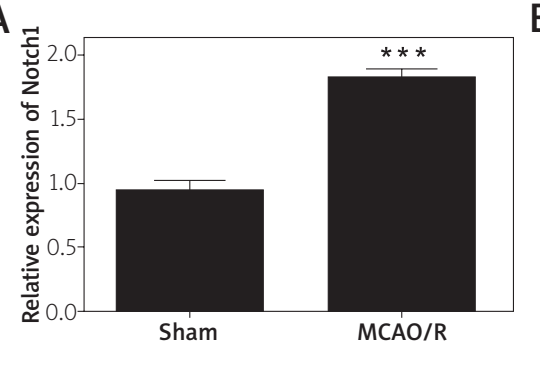

D
B

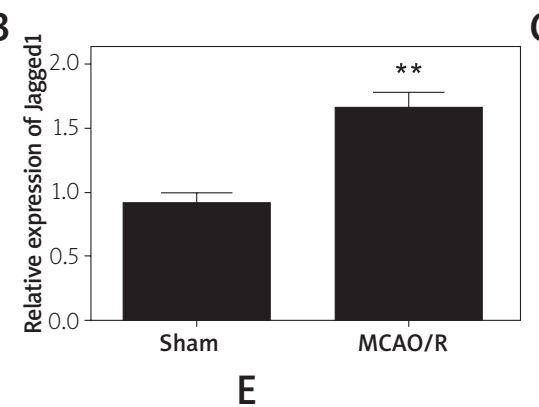

C

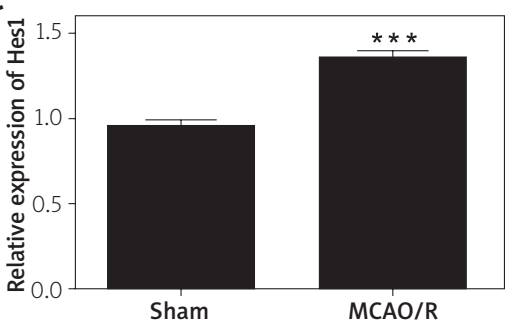

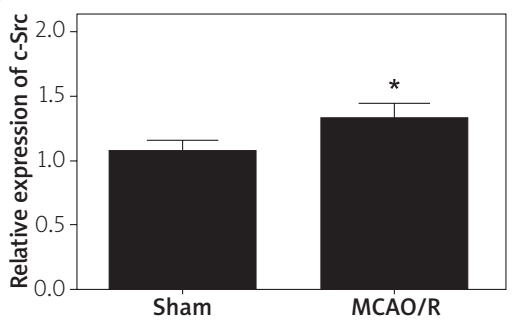

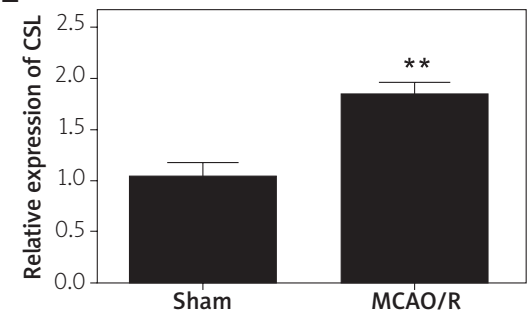

Fig. 1. The Notch1 signalling pathway is upregulated in a rat middle cerebral artery occlusion/reperfusion (MCAO/R) model. A) The expression levels of Notch1 in the sham operation group (Sham) and MCAO/R model group were detected by qPCR. B) The expression levels of Jagged1 in the sham operation group (Sham) and MCAO/R model group were detected by GPCR. C) The expression levels of Hes1 in the sham operation group (Sham) and MCAO/R model group were detected by qPCR. D) The expression of c-Src was detected by qPCR. E) The CSL expression level was detected by qPCR. ${ }^{*} p<0.05,{ }^{* *} p<0.01$, ${ }^{* * *} p<0.001$.

expression of CSL, a group of DNA-binding proteins which play a key role in the Notch signalling pathway, was up-regulated in the MCAO/R model group (Fig. 1E). Accordingly, changes in levels of inflammatory mediators were observed in the brain tissue of ischemia-induced rats. Levels of inflammatory mediators such as nuclear factor $\mathrm{\kappa B}$ (NF-kB), tumour necrosis factor $\alpha$ (TNF- $\alpha)$ and interleukin 6 (IL-6) in brain tissue homogenates were found to be higher in the MCAO/R model group than in the sham group (Fig. 2A-C).

\section{Decreased proliferation and increased apoptosis in the OGD/R neural stem cell model}

To further investigate the pathogenesis of cerebral ischemia, an in vitro cell model was constructed. The results of cell proliferation assay showed that the proliferation capacity of cells in the OGD/R group was decreased compared to the control (Fig. 3A, B). Moreover, cell migration experiments also showed a decreased migration capacity of neural stem cells after $\mathrm{OGD/R}$ treatment compared to the control
A

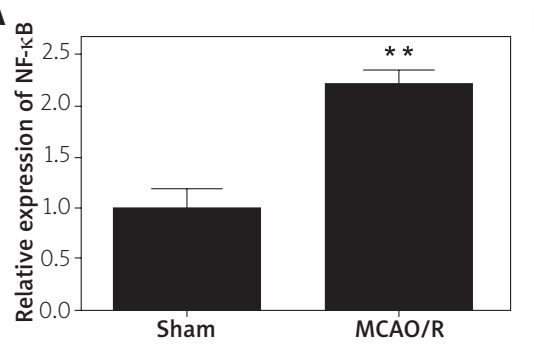

B

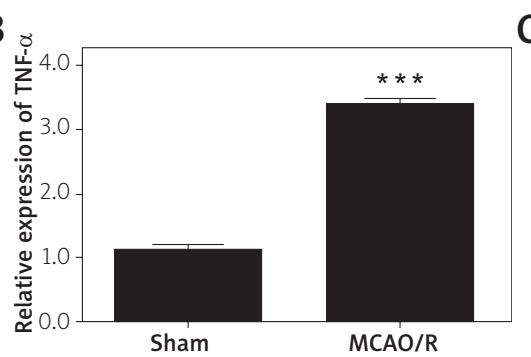

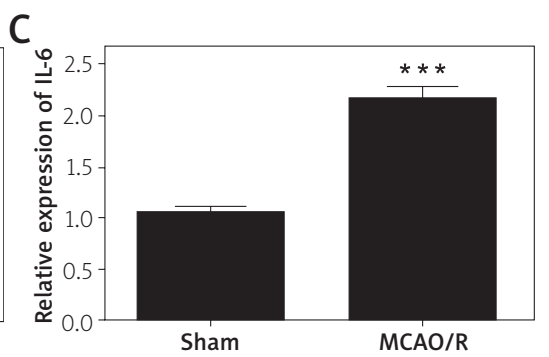

Fig. 2. The $M C A O / R$ model group increased levels of inflammatory factors. A) The expression of NF- $\kappa B$ was detected in the sham operation group (Sham) and MCAO/R model group. B) The expression of TNF- $\alpha$ was detected in the sham operation group (Sham) and MCAO/R model group. C) The expression of IL-6 was detected in the sham operation group (Sham) and MCAO/R model group. ${ }^{* *} p<0.01,{ }^{* *} p<0.001$. 
A

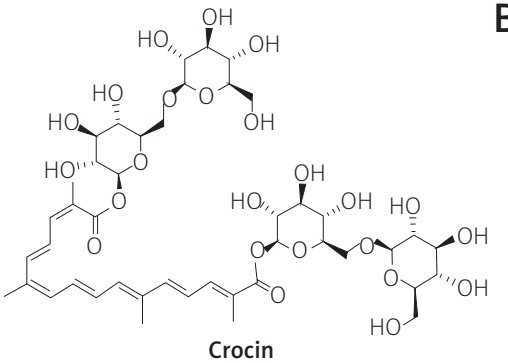

B

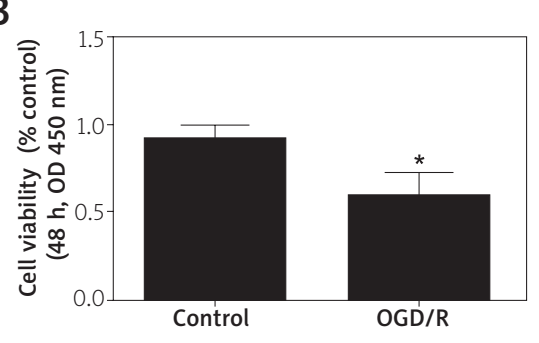

C

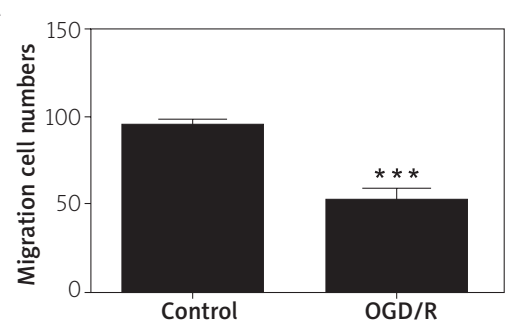

D
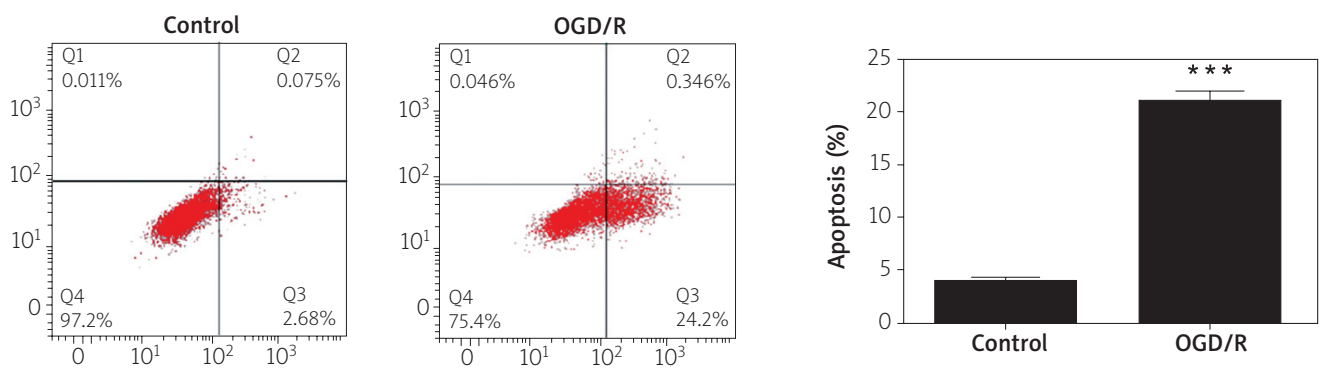

$E$
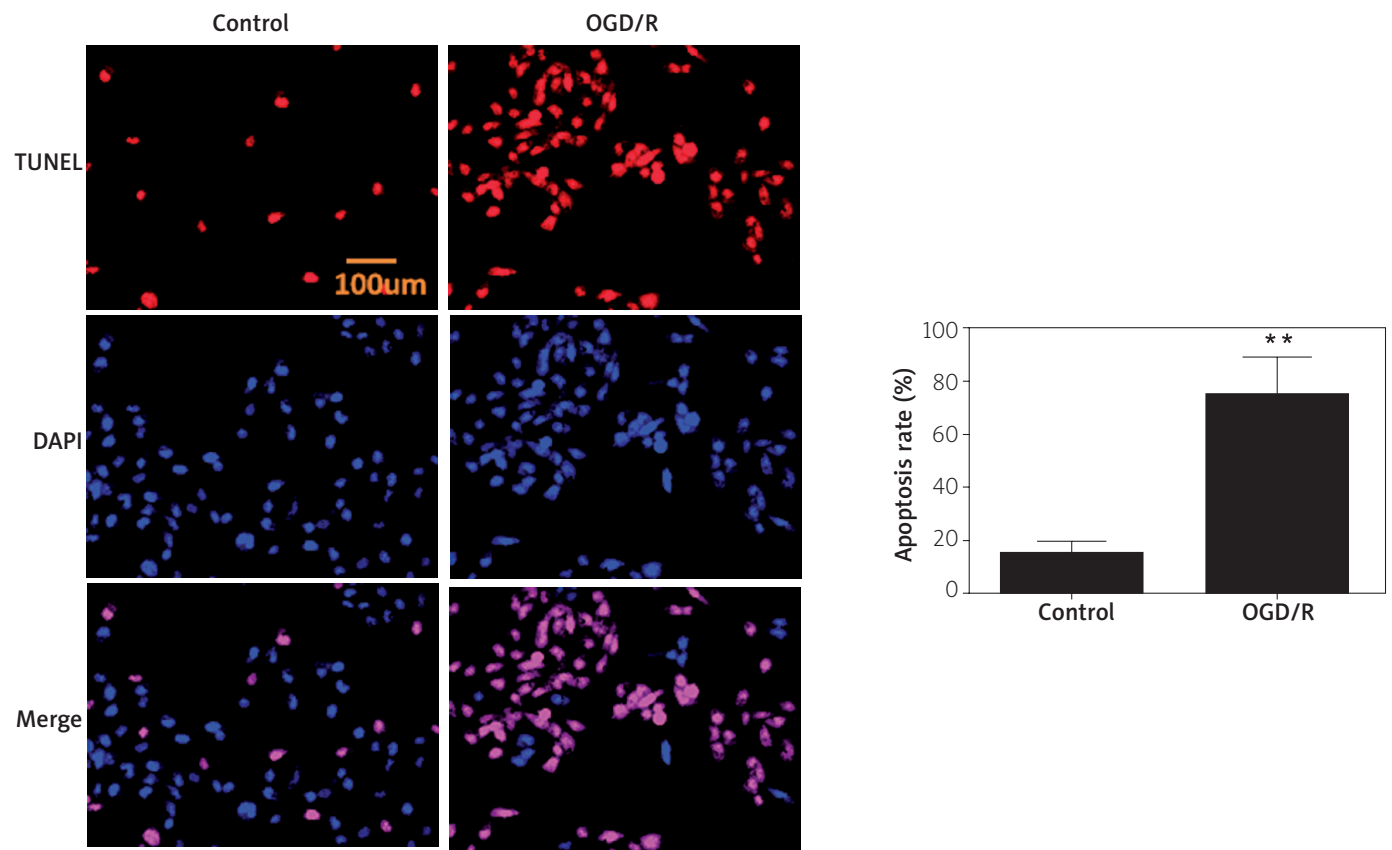

$\mathrm{F}$
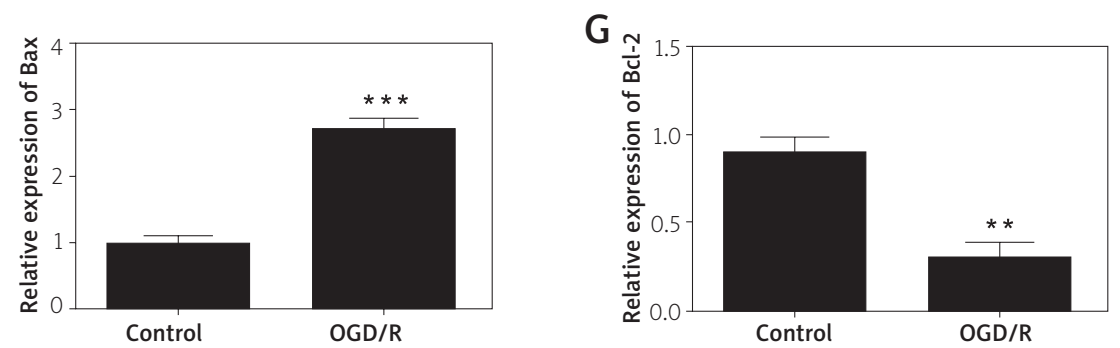

Fig. 3. The glucose deprivation/reoxygenation (OGD/R) neural stem cell model has reduced proliferation and increased apoptosis. A) The molecular structure of crocin. B) Cell proliferation ability was measured after different treatments. C) Cell migration was measured after different treatments. D) Flow cytometry to detect apoptosis. E) Apoptosis was detected by TUNEL staining. F) qPCR detection of Bax expression.

G) qPCR detection of Bcl-2 expression. ${ }^{*} p<0.05,{ }^{* *} p<0.01,{ }^{* \star *} p<0.001$. Magnification 200x. 
(Fig. 3C). To detect cell apoptosis we utilized the flow cytometry technique and our results showed that, compared with the control group, the apoptosis rate of neural stem cells increased after OGD/R treatment (Fig. 3D). TUNEL staining indicated a consistent finding observed in flow cytometry analysis. After OGD/R treatment, the apoptosis of neural stem cells increased (Fig. 3E). We therefore sought to detect the expression level of apoptosis-related protein Bax, and the data showed that, compared with the control group, the expression level of Bax was increased after OGD/R treatment (Fig. 3F). In addition, the level of anti-apoptotic protein bcl-2 was reduced in the OGD/R group (Fig. 3G).
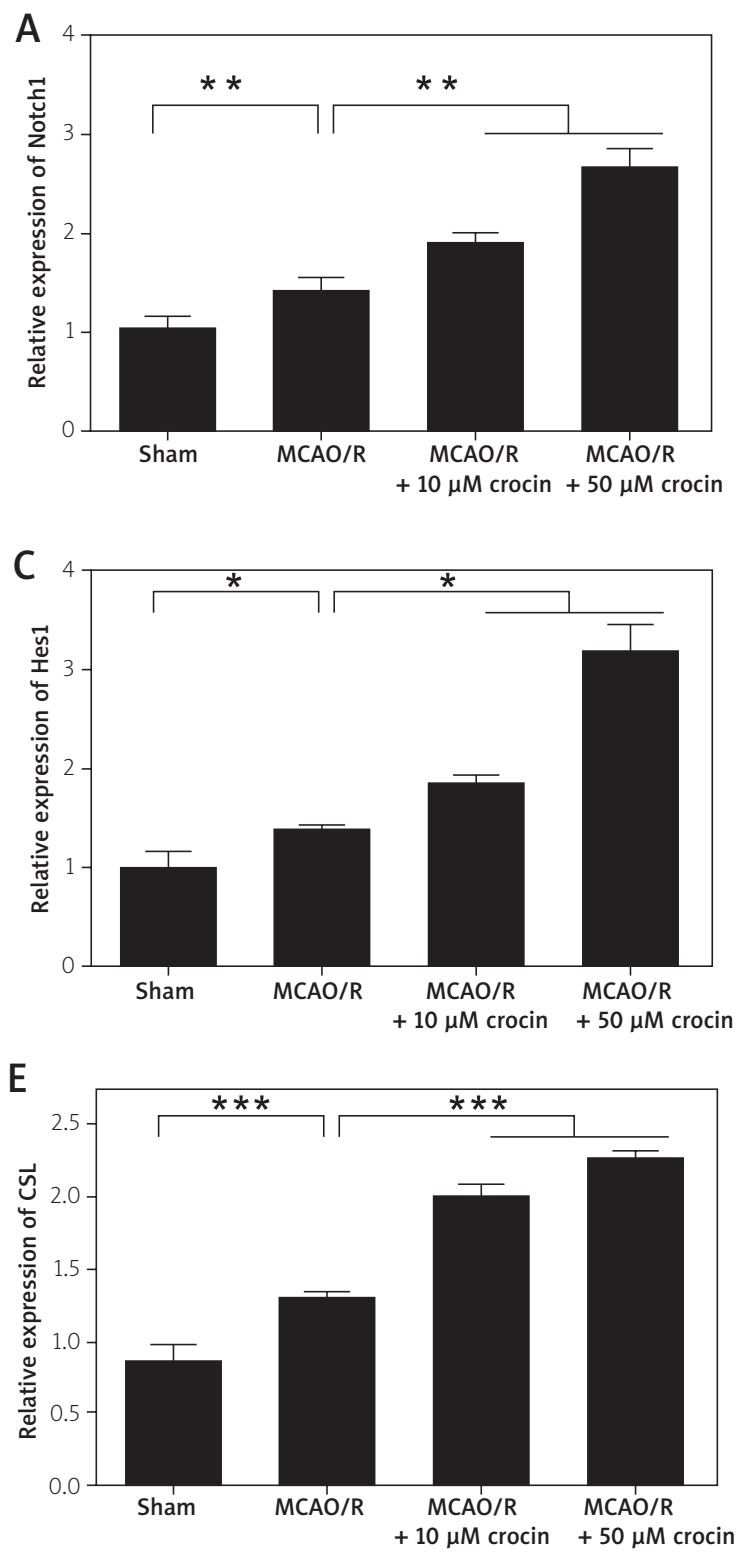

\section{Crocin activates the Notch1 signalling pathway in the MCAO/R model and inhibits inflammatory cytokines}

To understand the potential therapeutic effect of crocin on cerebral ischemia and its mechanism of action, we sought to determine the alterations of Notch1 in the sham group and model group after administration of crocin in our MCAO/R model. Our results showed that, compared with the control group, the expression level of Notch1 in the MCAO/R model group was elevated (Fig. 4A), and the Notch pathway could be further activated after administration of crocin. Moreover, our data demonstrated that
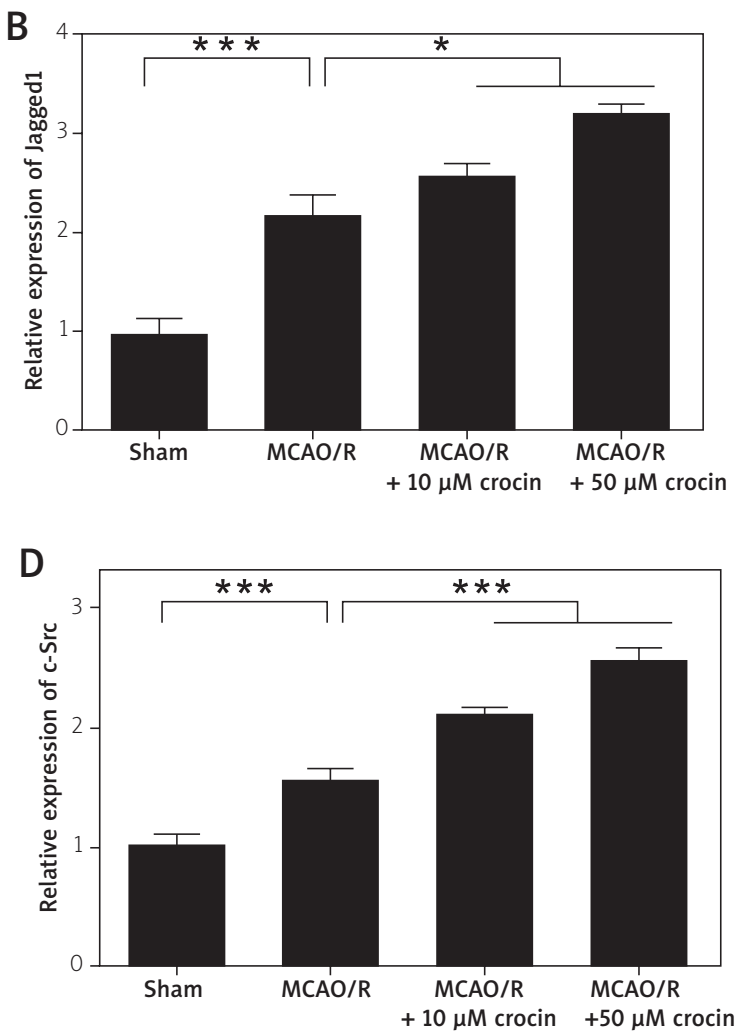

Fig. 4. Crocin activates the Notch1 signalling pathway in the MCAO/R model. A) Detection of Notch1 expression in different treatment groups. B) The Jagged1 expression level was measured after different treatments. C) The Hes1 expression was measured after different treatments. D) The c-Src expression level was measured after different treatments. E) The CSL expression level was measured after different treatments. ${ }^{*} p<0.05,{ }^{* *} p<0.01,{ }^{* * *} p<0.001$. 
A

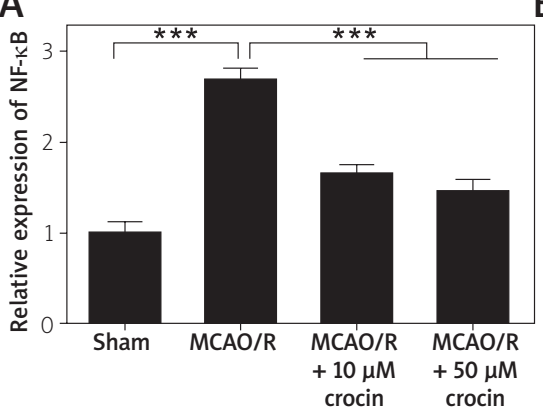

B

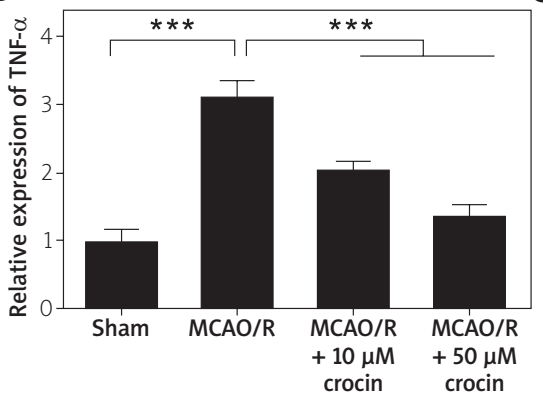

C

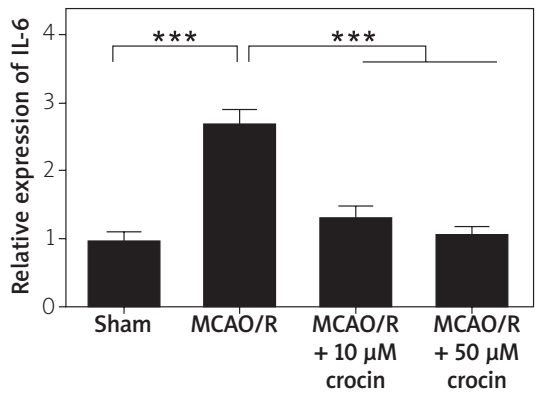

Fig. 5. Crocin inhibits inflammatory factors in the MCAO/R model group. A) Detection of NF-kB expression after different treatments. The expression level of NF-KB was decreased in the crocin treated group. B) Detection of TNF- $\alpha$ expression after different treatments. The expression level of TNF- $\alpha$ was decreased in the crocin treated group. C) Detection of IL-6 expression after different treatments. The expression level of IL- 6 was decreased in the crocin treated group. ${ }^{\star \star \star} p<0.001$.

the MCAO/R model induced an increased expression of Jagged1 (Fig. 4B) that can be further promoted by the administration of crocin. Next, similar to Notch1, we found that the variation trend of Hes1 and c-Src was also changed accordingly (Fig. 4C, D). Compared with the control group, the expression level of CSL was up-regulated in the MCAO/R model group (Fig. 4E), while the expression level of CSL could be further up-regulated. The above data illustrated that the expressions of Notch1 and Jagged1 in the model group were higher than those in the sham group. These results indicated that Notch signalling pathway may be involved in the proliferation of neural stem cells after cerebral ischemia. And our findings are consistent with the previous publications that glycoside activates the Notch pathway in addition to cerebral ischemia.

Levels of inflammatory mediators NF-KB, TNF- $\alpha$, and IL- 6 were observed in the brain tissue of ischemia-induced rats compared to the control group. In rats treated with safflower, the levels of NF-kB, TNF- $\alpha$ and IL- 6 in brain homogenate in the treatment group were dose-dependently reduced (Fig. 5A-C) compared with those in the model group.

\section{Crocin protects glucose deprivation/ reoxygenation (OGD/R) neural stem cells}

Finally, to further discover the protective effect of crocin on neural stem cells, we evaluated the effect of crocin on the proliferation and migration of neural stem cells utilizing the cell model constructed in vitro. Results of the cell proliferation experiment showed that compared with the control group, the proliferation ability of cells in the OGD/R group was reduced, but the proliferation ability of cells was enhanced after treatment with crocin (Fig. 6A). Furthermore, cell migration experimental results also confirmed that the migration ability of neural stem cells was reduced after OGD/R treatment, but the cell migration ability was enhanced after crocin treatment (Fig. 6B). And we have once again applied flow cytometry analysis to detect cell apoptosis and found that crocin sufficiently attenuated the increased apoptosis rate in neural stem cells induced by OGD/R (Fig. 6C). TUNEL staining results were consistent with the results of flow cytometry apoptosis detection. After OGD/R treatment, apoptosis of neural stem cells increased, while apoptosis was inhibited after addition of crocin (Fig. 6D). We further detected the expression level of apoptosis-related protein Bax. And our results suggested an increased level of Bax after OGD/R treatment. Yet this effect can be inhibited by crocin (Fig. 6E). The expression of anti-apoptotic protein Bcl-2 was reduced in the OGD/R group, while the expression of $\mathrm{Bcl}-2$ could be up-regulated in the crocin on the west (Fig. 6F).

\section{Discussion}

Numerous studies have demonstrated that cerebral ischemia activates endogenous neural stem cells in the adult brain, and researchers have always wanted to understand the regulatory mechanisms of neural stem cells and how they may be applied in treating stroke patients $[26,31,34]$. The regulatory mechanisms of neural stem cells are complex and still not fully understood. Age, diet, exercise 

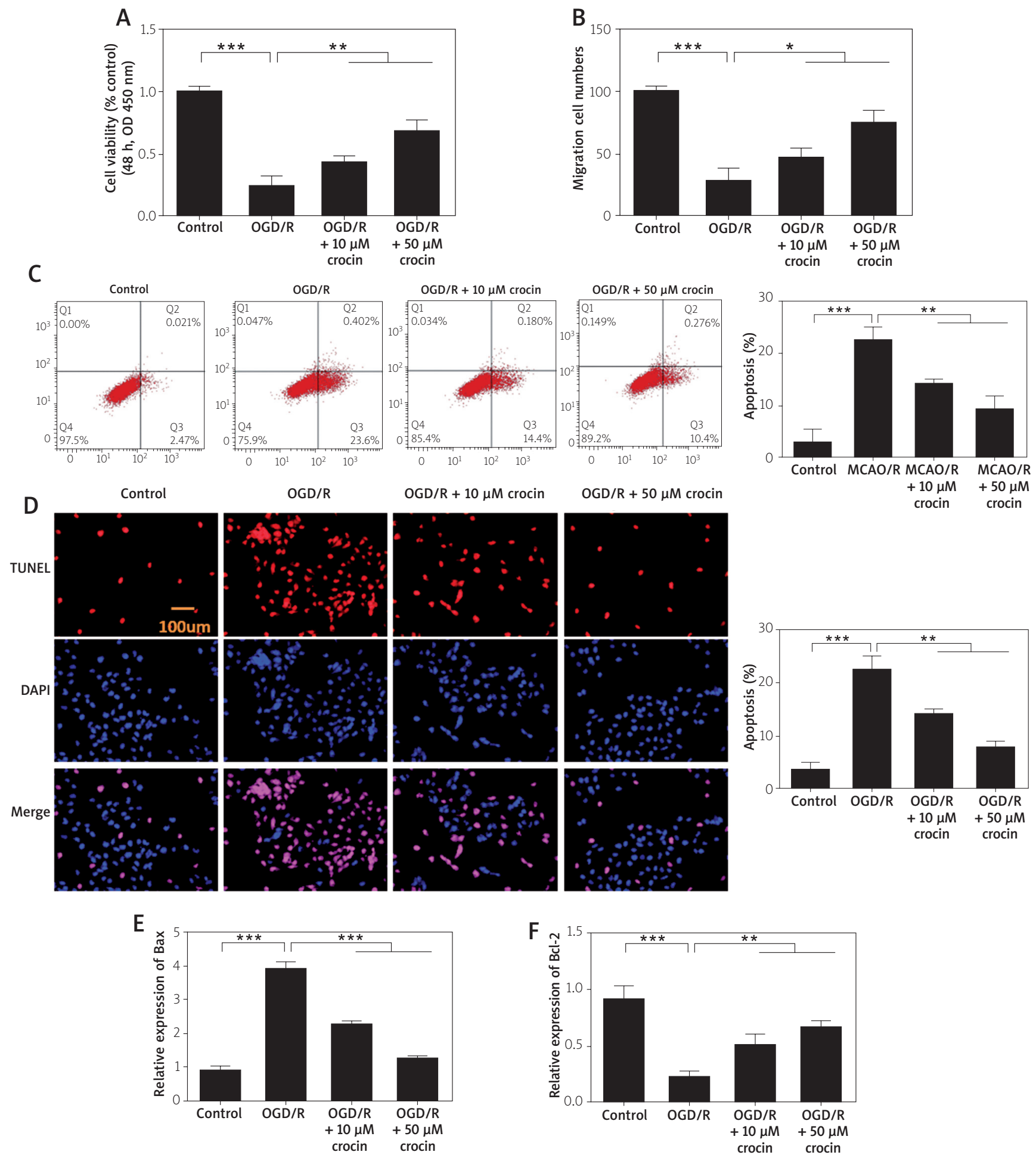

Fig. 6. Crocin contains glucose deprived/reoxygenated (OGD/R) neural stem cells. A) Detection of cell proliferation after different treatments. B) Detection of cell mobility after different treatments. C) Detection of apoptosis after different treatments. D) Detection of apoptosis after different treatments. E) After different treatments, qPCR was used to detect the expression of Bax. F) After different treatments, qPCR detected the expression of Bcl-2. ${ }^{*} p<0.05,{ }^{* *} p<0.01,{ }^{* * *} p<0.001$. Magnification $200 \times$. 
$[14,17,23]$ as well as signal transduction pathways and neurotrophic factors are all involved.

Studies suggest that neural regeneration can be promoted after cerebral ischemia. Iwai et al. [10] suggested that neural regeneration after cerebral ischemia can be divided into three stages: proliferation, migration and differentiation. The cell proliferation reached a peak on the $10^{\text {th }}$ day after cerebral ischemia. The cells that began to proliferate 20 days after ischemia expressed neural cell adhesion molecule (NCAM), and migrated to human granulosa from the subgranulosa region. However, it was not until 60 days after ischemia that the migrating cells turned into mature neurons. Sato et al. [23] observed that there was only a small amount of NCAM expression in SVZ in normal rats, and the number of older animals was slightly higher than that in the young group. After ischemia for $90 \mathrm{~min}$, the number of NCAM positive cells increased in the young group and reached a peak after 1 day, while the expression of NCAM was the highest in the old group after 3 days. This suggests that cerebral ischemia can promote migration of neural stem cells.

The Notch signalling pathway is known to maintain the cellular characteristics of NSCs and inhibit neurogenesis $[9,16,36]$. The reason why neural stem cells can continuously produce new nerve cells is precisely because of the regulation of Notch signalling pathway, so that the proliferation and differentiation of NSCs can maintain a stable equilibrium state. The Notch signalling pathway plays an important role in the multi-directional differentiation of NSCs $[5,8]$. In our particular study, animal experiments demonstrated that crocin activates the signal of Notch1 in brain ischemia-reperfusion injury tissues. Compared with the sham group, the expression levels of Notch1 were up-regulated in the model group and the administration group. Compared with the model group, the expression of Notch1 was more up-regulated after treatment with crocin. In this study, it was also found that the proliferation of neural stem cells increased significantly after oxyglycemic deprivation/reoxygenation, but the cell viability may be decreased and the apoptosis may be increased, which is consistent with the expression after ischemia-reperfusion in vivo. By activating Notch1, crocin can promote the survival of neurons, inhibit the release of inflammatory cytokines, and reduce the apoptosis of neural stem cells.
After ischemia-reperfusion, the generation of reactive oxygen species is the main cause of neuronal injury and even apoptosis. Improving the antioxidant capacity of cells can reduce the damage [5]. Studies have found that crocin has a strong antioxidant capacity, which can inhibit the production of oxygen free radicals and reduce cell damage [22,33]. There are also studies showing that the neuroprotective effect of crocin is to reduce the neuron damage caused by oxidative stress by inhibiting the activity of peroxidase and enhancing the activity of superoxide dismutase. In addition, in the serum-induced model of PC12 cell death, it was found that crocin can reduce the death of neurons and protect brain ischemia reperfusion injury by increasing glutathione reductase, decreasing sphingomyelinase activity and reducing the formation of ceramide [21].

\section{Conclusions}

In summary, this study demonstrated that crocin can protect neural populations after ischemia/reperfusion in MCAO/R model rats, and the mechanism of action may be related to the Notch1 signalling pathway. The results of in vitro cell experiments indicate that crocin may promote cell proliferation, increase cell migration, inhibit cell apoptosis, and promote neural regeneration. Therefore, the molecular mechanism of the neuroprotective effect of crocin is to promote the proliferation and migration of neural stem cells by activating Notch1. At the same time, it can inhibit the release of inflammatory factors and reduce the apoptosis of nerve cells caused by ischemia and hypoxia.

\section{Funding}

Heilongjiang Province Provincial Higher Education Fund Research Project (2019-KYYWF-0993); Heilongjiang Provincial Health Commission Research Project (2019-387); Heilongjiang Provincial Health and Family Planning Commission Research Project (2017-314).

\section{Disclosure}

The authors declare no conflict of interest.

\section{References}

1. Arvidsson A, Kokaia Z, Lindvall O. N-methyl-D-aspartate receptormediated increase of neurogenesis in adult rat dentate gyrus following stroke. Eur J Neurosci 2001; 14: 10-18. 
2. Astrup J, Siesjö BK, Symon L. Thresholds in cerebral ischemia the ischemic penumbra. Stroke 1981; 12: 723-725.

3. Borghese L, Dolezalova D, Opitz T, Haupt S, Leinhaas A, Steinfarz B, Koch P, Edenhofer F, Hampl A, Brüstle O. Inhibition of notch signaling in human embryonic stem cell-derived neural stem cells delays $\mathrm{G} 1 / \mathrm{S}$ phase transition and accelerates neuronal differentiation in vitro and in vivo. Stem Cells 2010; 28: 955-964.

4. Cai Q, Chen Z, Kong DK, Wang J, Xu Z, Liu B, Chen Q, Zhou J. Novel microcatheter-based intracarotid delivery approach for MCAO/R mice. Neurosci Lett 2015; 597: 127-131.

5. Chan $\mathrm{PH}$. Reactive oxygen radicals in signaling and damage in the ischemic brain. J Cerebr Blood F Met 2001; 21: 2-14.

6. Chen CY, Liao W, Lou YL, Li Q, Hu B, Wang Y, Deng ZF. Inhibition of Notch signaling facilitates the differentiation of humaninduced pluripotent stem cells into neural stem cells. Mol Cell Biochem 2014; 395: 291-298.

7. Chen L, Huang K, Wang R, Jiang Q, Wu Z, Liang W, Guo R, Wang $L$ Neuroprotective effects of cerebral ischemic preconditioning in a rat middle cerebral artery occlusion model: the role of the Notch signaling pathway. Biomed Res Int 2018; 2018: 8168720.

8. Guan J, Wei X, Qu S, Lv T, Fu Q, Yuan Y. Osthole prevents cerebral ischemia-reperfusion injury via the Notch signaling pathway. Biochem Cell Biol 2017; 95: 459-467.

9. Huang G, Cao X, Zhang X, Chang H, Yang Y, DU W, Wilson JX. Effects of soybean isoflavone on the notch signal pathway of the brain in rats with cerebral ischemia. J Nutr Sci Vitaminol 2009; 55: 326-331.

10. Iwai M, Sato K, Omori N, Nagano I, Manabe Y, Shoji M, Abe K. Three steps of neural stem cells development in gerbil dentate gyrus after transient ischemia. J Cerebr Blood F Met 2002; 22: 411-419.

11. Jin K, Minami M, Lan JQ, Mao XO, Batteur S, Simon RP, Greenberg DA. Neurogenesis in dentate subgranular zone and rostral subventricular zone after focal cerebral ischemia in the rat. Proc Natl Acad Sci U S A 2001; 98: 4710-4715.

12. Karakani AM, Riazi G, Ghaffari SM, Ahmadian S, Mokhtari F, Firuzi MJ, Bathaie SZ. Inhibitory effect of corcin on aggregation of 1N/4R human tau protein in vitro. Iran J Basic Med Sci 2015; 18: 485.

13. Kee N, Preston E, Wojtowicz J. Enhanced neurogenesis after transient global ischemia in the dentate gyrus of the rat. Exp Brain Res 2001; 136: 313-320.

14. Kempermann G, van Praag H, Gage FH. Activity-dependent regulation of neuronal plasticity and self repair. Prog Brain Res 2000; $127: 35-48$

15. Kong R, Zhang Y, Zhang S, Liu M, Sun W, Xing Y, Guan Y, Han C, Liu Z. Protective effect of ethanol extracts of the Chinese caterpillar mushroom, Ophiocordyceps sinensis (Ascomycetes), on the experimental middle cerebral artery occlusion/reperfusion (MCAO/R) model. Int J Med Mushrooms 2015; 17: 997.

16. LeComte MD, Shimada IS, Sherwin C, Spees JL. Notch1-STAT3ETBR signaling axis controls reactive astrocyte proliferation after brain injury. Proc Natl Acad Sci USA 2015; 112: 8726-8731.

17. Lee J, Duan W, Long JM, Ingram DK, Mattson MP. Dietary restriction increases the number of newly generated neural cells, and induces BDNF expression, in the dentate gyrus of rats. J Mol Neurosci 2000; 15: 99-108.
18. Li S, Zhang X, Wang Y, Ji H, Du Y, Liu H. DAPT protects brain against cerebral ischemia by down-regulating the expression of Notch 1 and Nuclear factor kappa B in rats. Neurol Sci 2012; 33: $1257-1264$

19. Liang W, Lin C, Yuan L, Chen L, Guo P, Li P, Wang W, Zhang X. Preactivation of Notch1 in remote ischemic preconditioning reduces cerebral ischemia-reperfusion injury through crosstalk with the NF-אB pathway. J Neuroinflammation 2019; 16: 181.

20. Liao LY, Lau BWM, Sánchez-Vidaña DI, Gao Q. Exogenous neural stem cell transplantation for cerebral ischemia. Neural Regen Res 2019; 14: 1129.

21. Ochiai T, Ohno S, Soeda S, Tanaka H, Shoyama Y, Shimeno H. Crocin prevents the death of rat pheochromyctoma (PC-12) cells by its antioxidant effects stronger than those of $\alpha$-tocopherol. Neurosci Lett 2004; 362: 61-64.

22. Ordoudi SA, Befani CD, Nenadis N, Koliakos GG, Tsimidou MZ. Further examination of antiradical properties of Crocus sativus stigmas extract rich in crocins. J Agr Food Chem 2009; 57: 3080-3086.

23. Sato K, Hayashi T, Sasaki C, Iwai M, Li F, Manabe Y, Seki T, Abe K. Temporal and spatial differences of PSA-NCAM expression between young-adult and aged rats in normal and ischemic brains. Brain Res 2001; 922: 135-139.

24. Takarada-lemata M, Yoshikawa A, Ta HM, Okitani N, Nishiuchi T, Aida Y, Kamide T, Hattori T, Ishii H, Tamatani T. N-myc downstream-regulated gene 2 protects blood-brain barrier integrity following cerebral ischemia. Glia 2018; 66: 1432-1446.

25. Tian T, Zhang HX, He CP, Fan S, Zhu YL, Qi C, Huang NP, Xiao ZD, Lu ZH, Tannous BA. Surface functionalized exosomes as targeted drug delivery vehicles for cerebral ischemia therapy. Biomaterials 2018; 150: 137-149.

26. Tu XK, Yang WZ, Shi SS, Wang CH, Chen CM. Neuroprotective effect of baicalin in a rat model of permanent focal cerebral ischemia. Neurochem Res 2009; 34: 1626-1634.

27. Turlova E, Feng ZP, Sun HS. The role of TRPM2 channels in neurons, glial cells and the blood-brain barrier in cerebral ischemia and hypoxia. Acta Pharmacol Sin 2018; 39: 713-721.

28. Wang Y, Cai Z, Luo Y, Gong J. Influence of edaravone on Notch1 and nuclear factor-kappaB in rats with cerebral ischemia/reperfusion injury. Neural Regen Res 2008; 3: 1342-1347.

29. Yin D, Zhang JH. Delayed and multiple hyperbaric oxygen treatments expand therapeutic window in rat focal cerebral ischemic model. Neurocrit Care 2005; 2: 206-211.

30. Zhang HP, Sun YY, Chen XM, Yuan LB, Su BX, Ma R, Zhao RN, Dong $\mathrm{HL}$, Xiong $\mathrm{L}$. The neuroprotective effects of isoflurane preconditioning in a murine transient global cerebral ischemiareperfusion model: The role of the notch signaling pathway. Neuromol Med 2014; 16: 191-204.

31. Zhang H, Sun X, Xie Y, Tian F, Hu H, Tan W. Isosteviol sodium inhibits astrogliosis after cerebral ischemia/reperfusion injury in rats. Biol Pharm Bull 2018; 41: 575-584.

32. Zhang XX, Yin LK, Hao XZ, Tian JQ, Li CC, Feng XY, Yang YM. Imaging the transformation of ipsilateral internal capsule following focal cerebral ischemia in rat by diffusion kurtosis imaging. J Stroke Cerebrovasc 2017; 26: 42-48.

33. Zheng YQ, Liu JX, Wang JN, XU L. Effects of crocin on reperfusion-induced oxidative/nitrative injury to cerebral microvessels after global cerebral ischemia. Brain Res 2007; 1138: 86-94. 
34. Zheng Y, Song T, Zhang L, Wei N. Immunomodulatory effects of T helper 17 cells and regulatory $T$ cells on cerebral ischemia. J Biol Reg Homeos Ag 2018; 32: 29-35.

35. Zhong W, Sun B, Gao W, Qin Y, Zhang H, Huai L, Tang Y, Liang Y, He L, Zhang X, Tao H, Chen S, Yang W, Yang L, Liu Y, Liu H, Zhou H, Sun T, Yang C. Salvianolic acid A targeting the transgelin-actin complex to enhance vasoconstriction. EBioMedicine 2018; 37 : 246-258.

36. Zhong W, Yang W, Qin Y, Gu W, Xue Y, Tang Y, Xu H, Wang H, Zhang C, Wang C. 6-Gingerol stabilized the p-VEGFR2/ VE-cadherin/ $\beta$-catenin/actin complex promotes microvessel normalization and suppresses tumor progression. J Exp Clin Canc Res 2019; 38: 285. 